\title{
Modeling and research of the process of preparing the surface of a part for thermal spray coating
}

\author{
Aleksandr Novikov, Aleksei Rodishev*, Andrei Gorin, and Maria Tokmakova \\ Orel State University named after I. S. Turgenev, Komsomolskaya st. 95, 302026, Orel, Russia
}

\begin{abstract}
The article presents an analysis of ways to increase the adhesive strength of antifriction coating. A rational method for the mechanical formation of surfaces is proposed. The calculation of the workpiece based on the finite element method has been carried out. The theoretical dependences of metal lifting on the depth of the rolling roller are obtained. The results of a field experiment are presented. A comparative analysis of theoretical and practical results of the research has been carried out. Recommendations are given for the further use of the net rolling method with a pre-cut "ragged thread".
\end{abstract}

\section{Introduction and Problem formulation}

One of the most rational ways to increase the wear resistance of plain bearings is the use of bimetallic bushings with a coating of antifriction copper-based powders applied to the inner surface. The main parameter that characterizes the performance of antifriction coatings is adhesion strength. The adhesion strength is determined by the interfacial interaction and the structure of the boundary layer. Previous research work on the study of the performance of parts with coatings has convincingly shown that the main reason for their destruction is an insufficient connection between the coating material and the base [1, 2, 3, 4].

Adhesion strength can be increased by forming a special relief on the base surface $[5,6]$. Creation of a developed relief on the surface of a part is a widespread and effective way of increasing the adhesion strength of the filler material to the surface of the part during spraying. The use of methods of mechanical shaping of surfaces allows you to increase the roughness or create a pronounced relief. One of the most rational types of applied reliefs for internal surfaces is a mesh profile obtained by rolling. The greatest effect is obtained by using a mesh profile in combination with a pre-cut «ragged thread» $[7,8]$. However, the use of this progressive technology in industry is constrained by the fact that oblique mesh rolling on inner cylindrical surfaces has hardly been studied. In particular, there are no recommendations for determining the diameter of the workpiece for rolling, deformation forces and other technological parameters.

\footnotetext{
* Corresponding author: rodfox@yandex.ru
} 


\section{Theoretical part}

To determine the diameter of the workpiece for oblique mesh rolling, it is necessary to identify the nature of the metal redistribution along the height during plastic deformation of the base. Since the metal during oblique mesh rolling moves in all three directions, it is necessary to consider a volumetric problem to determine the diameter of the workpiece. The diameter of the workpiece is calculated based on the principle of equality of the metal volumes before and after deformation. The obtained mathematical dependences do not take into account the redistribution of metal in space due to filling the groove of the knurling roller. Therefore, in order to obtain a more complete understanding of the process that occurs during the plastic deformation of the base during rolling, it is necessary to simulate the process of mesh rolling along the thread on the inner cylindrical surface using the finite element method (FEM). From modern software products based on FEM, the most preferred for rolling simulation is the use of the DEFORM system $[9,10]$.

Modeling was carried out in a volumetric formulation of the problem (Fig. 1). In accordance with the calculated data, the inner diameter of the workpiece before rolling onto the inner surface of the profile was $\varnothing 91.8 \mathrm{~mm}$. The thread was made in the form of a spiral groove in accordance with its geometric dimensions. To reduce the number of FE in order to simplify the calculations and obtain more accurate results, the sleeve width was taken equal to $4 \mathrm{~mm}$, modeling was performed for $1 / 2$ part. When modeling, the workpiece was taken rigidly plastic, the tool was ideally rigid. The mechanical properties of the workpiece material (Steel 45) were set by a multilinear hardening curve. The normalized Cockcroft Leitem criterion $[11,12]$ was used as a criterion for material failure.

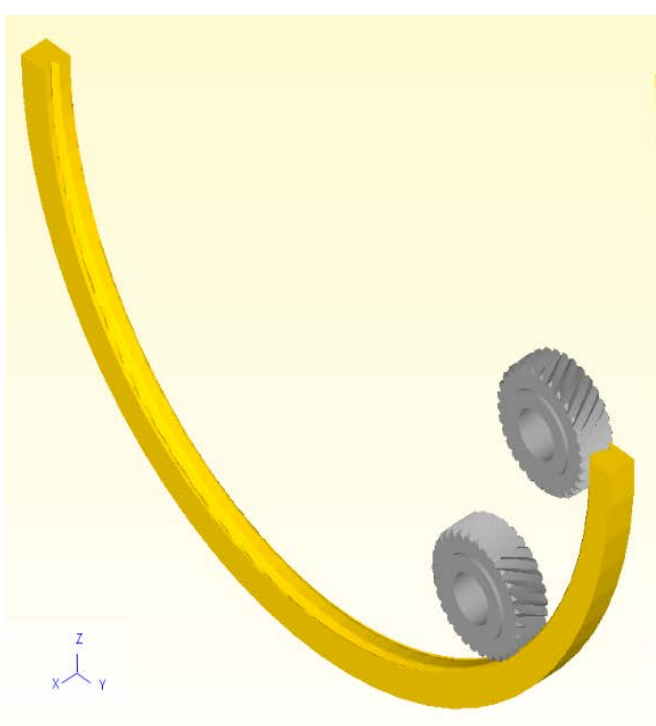

before deformation

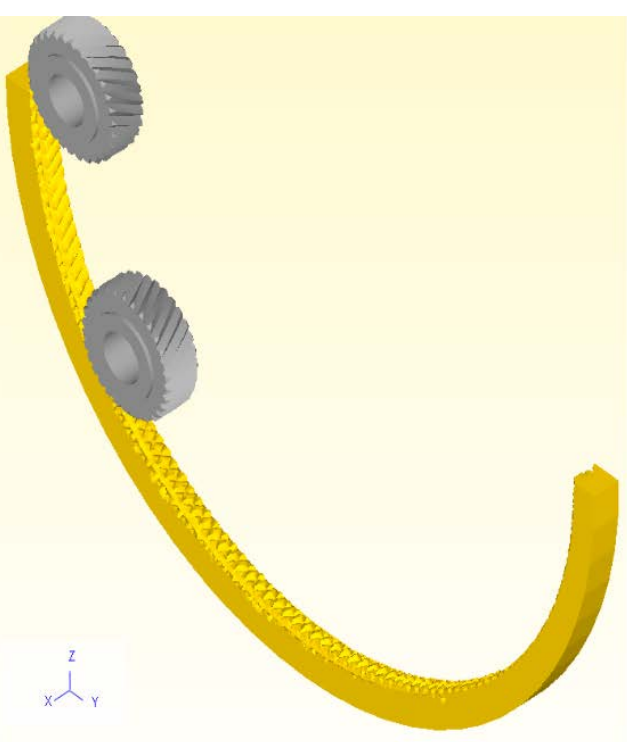

after deformation

Fig. 1. Geometric model of oblique mesh rolling.

When dividing the model into finite elements, a tetrahedral Lagrangian mesh was used. The number of elements was chosen so that the side length of the smallest element was 3 times less than the radius of the root of the rolled thread. During modeling, the workpiece remained motionless, and all movements were imparted to the tool. The tool rolled along the workpiece without slipping. The rolling speed was $20 \mathrm{~m} / \mathrm{min}$. When carrying out a computational experiment, the depths of the knurling rollers were varied $\mathrm{h}=0.4 \ldots 0.8 \mathrm{~mm}$; 
the pitch of the "torn" thread $\mathrm{P}=2 \mathrm{~mm}$ and the pitch of the knurling roller $\mathrm{t}=1.6 \mathrm{~mm}$. The theoretical results of metal distribution depending on the depth of the knurling rollers are shown in Figure 2.
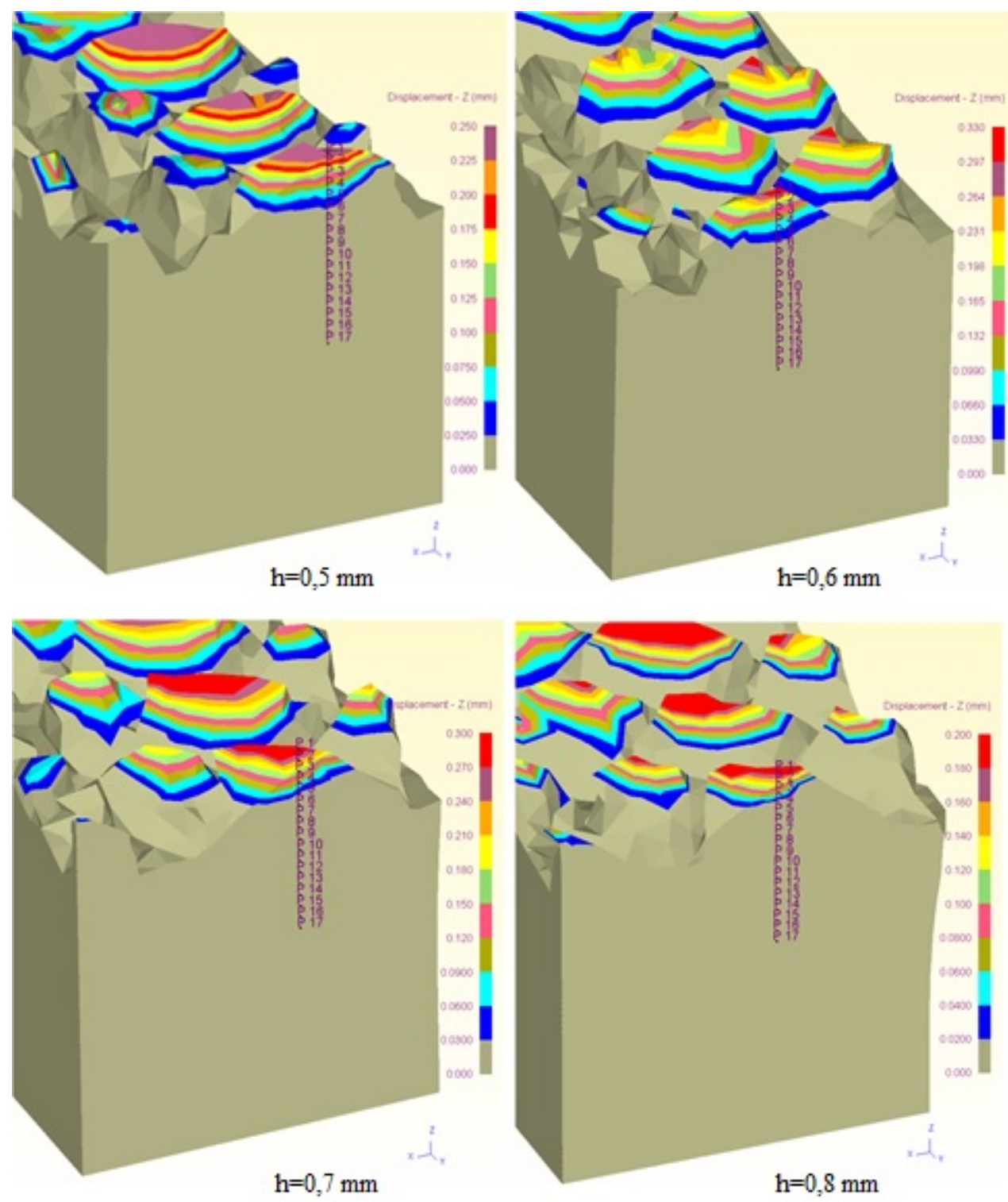

Fig. 2. Increment and distribution of metal depending on the depth of the knurling rollers $h$

Based on the results of the theoretical calculations obtained after modeling the process of oblique mesh rolling, a graph of the dependence of the increase in metal on the deepening of the knurling roller was built (Figure 3). 


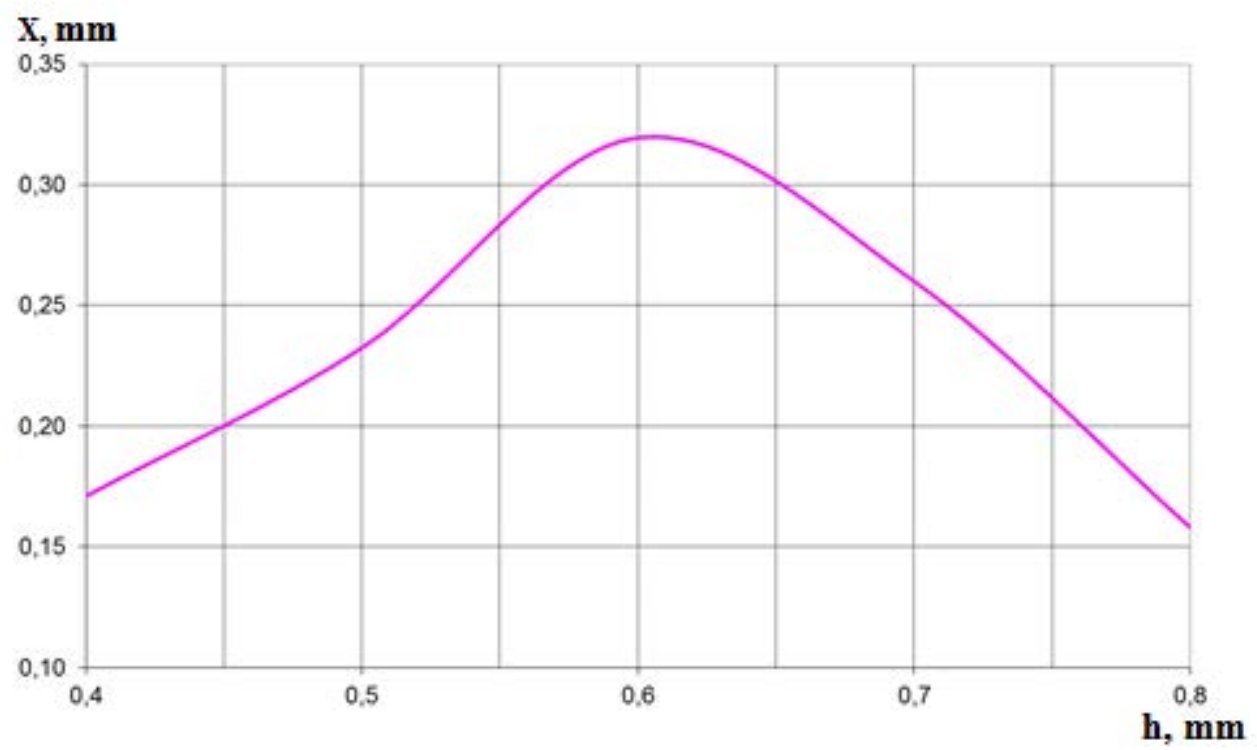

Fig. 3. Graph of the dependence of the rise of metal $x$ on the depth of the knurling roller $h$

\section{Experimental studies}

The adequacy of the simulation results in DEFORM was confirmed by a full-scale experiment. For the implementation of the process of oblique mesh rolling on the inner surfaces, a design of a self-aligning knurling head has been developed. The experimental determination of the rise of the metal was carried out by optical measurement of the geometric parameters of the surface after rolling, using the projection (shadow) method on a universal measuring microscope UIM-21 (Fig. 4, 5).

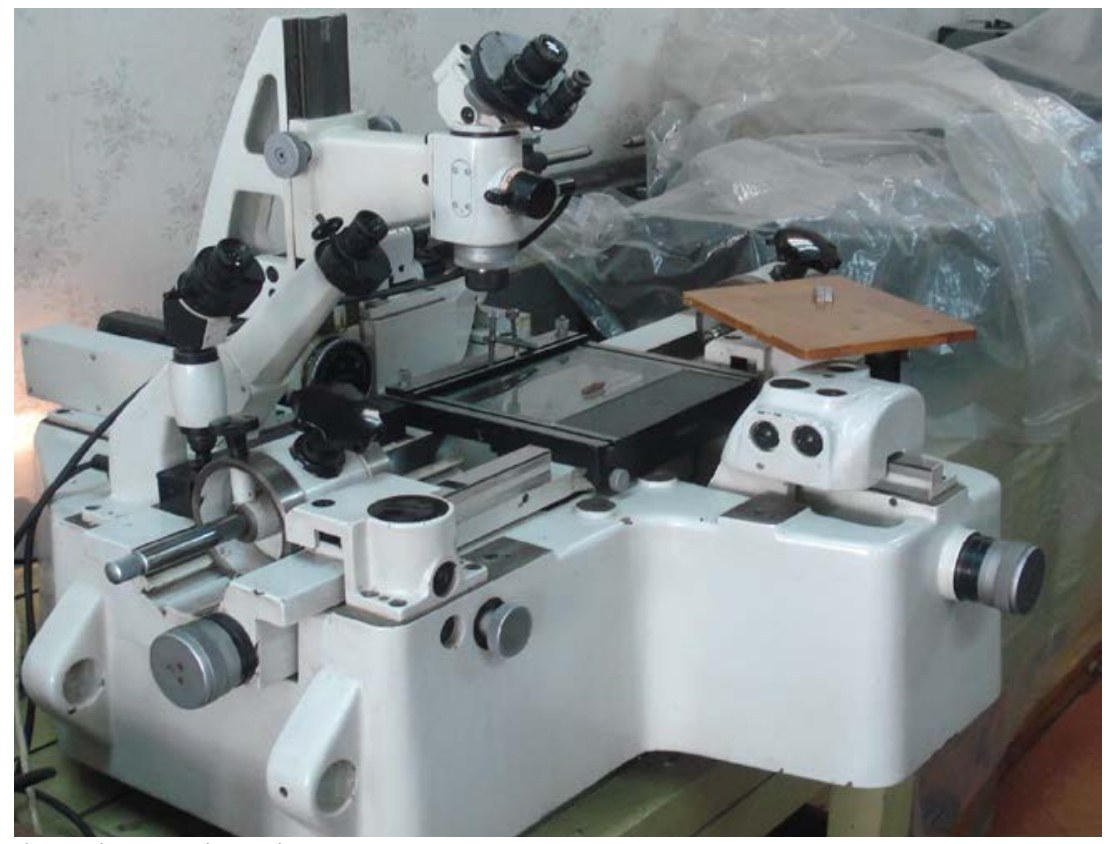

Fig. 4. Universal measuring microscope UIM-21 


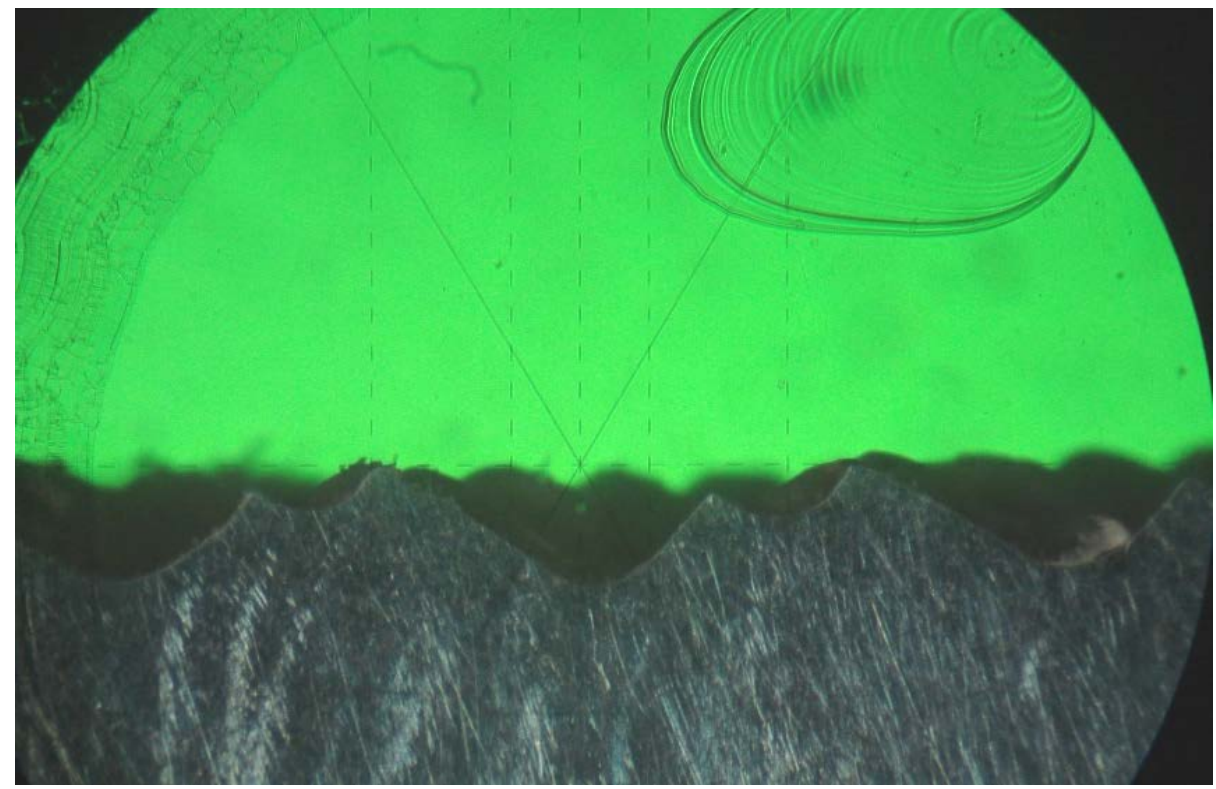

Fig. 5. Measurement by the projection (shadow) method of the geometric parameters of the surface after rolling

Based on the results of practical calculations, a graph of the dependence of the increase in metal on the depth of the knurling roller was obtained. Let's combine the resulting graph with the theoretical dependence (Figure 6).

\section{$\mathbf{x}, \mathbf{m m}$}

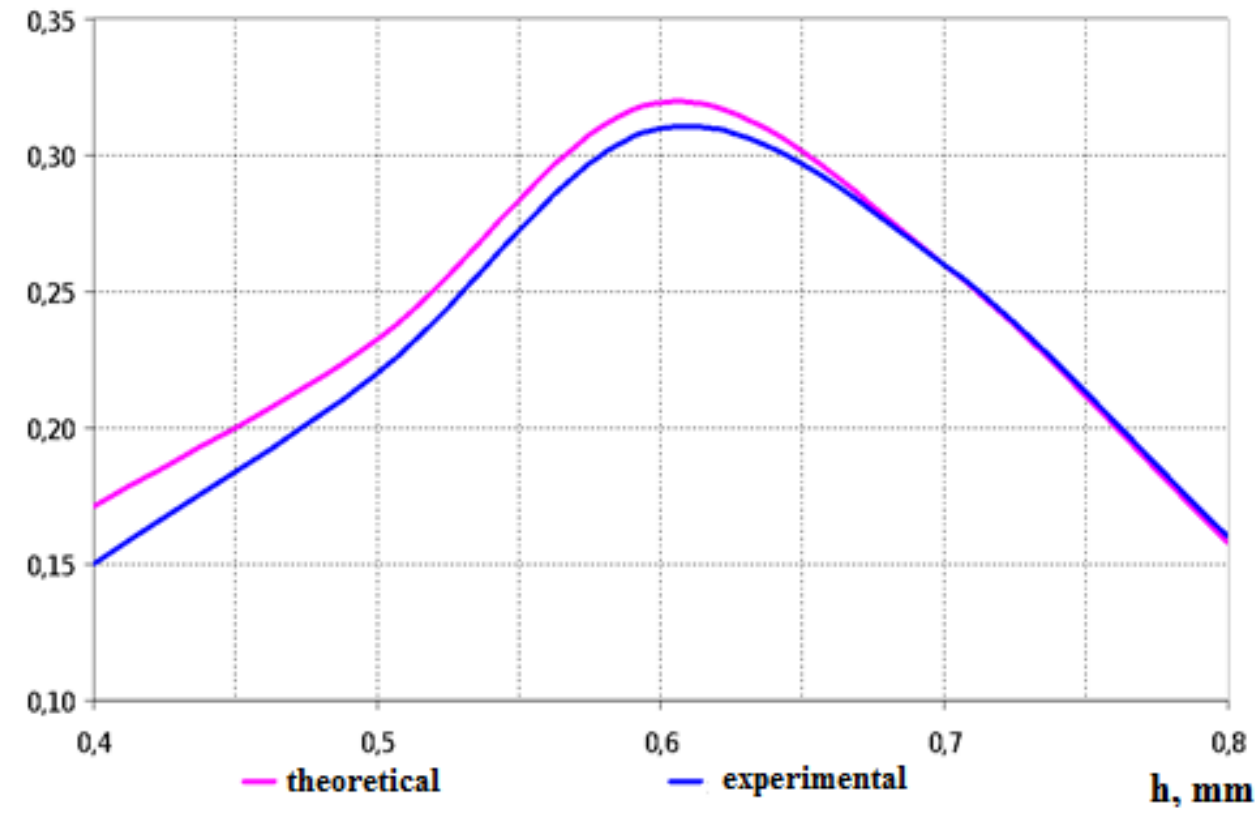

Fig. 6. Graphs of the dependence of the rise of metal $x$ on the depth of the knurling roller $h$ 


\section{Conclusions}

As a result of the research, it was found that the most rational method for calculating the parameters of the workpiece for oblique mesh rolling is the finite element method. A graph of the dependence of the rise of metal $\mathrm{x}$ on the depth of the knurling roller $\mathrm{h}$ is obtained. The deviation of the values of metal rise obtained as a result of computational and full-scale experiments does not exceed $5 \%$.

The application of the proposed method of volumetric modeling allows obtaining objective data for calculating the optimal modes of mechanical surface treatment.

\section{References}

1. Yu.S. Borisov, Yu.A. Kharlamov, Thermal gas coatings from powder materials. (Naukova Dumka, 1987)

2. M.Kh. Shoroshorov, Yu.A. Kharlamov, Physicochemical bases of denotation - gas spraying of coatings. ( Nauka, 1978)

3. S.Yu. Sharivker, E.A. Astakhov, A.P. Gaida, Influence of the flight speed of sprayed particles on the adhesion strength of sprayed coatings. Physics and Chemistry of Materials Processing, №5,(1984)

4. A. Hasui, O. Morichaki, Surfacing and spraying (Mechanical Engineering, 1985)

5. VN Korenev, Increasing the adhesion strength of gas-flame coatings with the base by rolling on the thread of the lock profile.(Orel, 2006)

6. V.P. Lyalakin, V.P. Ivanov, V.M. Konstantinov Restoration of machine parts. (Mechanical Engineering, 2003)

7. A.Yu. Rodichev, V.N. Khromov, V.N. Korenev, Method of preparation of product surface for spraying (RF patent 2400312, 2010)

8. V.N. Khromov, V.N. Korenev, A.Yu. Rodichev, Technology of surface preparation of a product for spraying. Fundamental and applied problems of engineering and technology, No. 2 (280) (2010)

9. A.V. Kirichek, A.N. Afonin, Investigation of the stress-strain state of a threadrolling tool and workpiece by the finite element method, STIN., No. 7. (2007)

10. A.N. Afonin, Assessment of thread hardening based on the results of computer modeling of thread rolling (Engineering systems, 2011)

11. A.L. Vorontsov, Comparison of the application of the finite element method and analytical methods for solving pressure processing problems (Bulletin of mechanical engineering, 2003)

12. A.O. Chernavsky, Finite element method. Basics of practical application, Engineering journal, No. 11, (2003) 